\title{
Flare Ribbon Expansion and Energy Release Rate
}

\author{
Ayumi Asai ${ }^{1}$, Takaaki Yokoyama ${ }^{2}$, Masumi Shimojo ${ }^{3}$, \\ Satoshi Masuda ${ }^{4}$, and Kazunari Shibata ${ }^{1}$ \\ ${ }^{1}$ Kwasan and Hida Observatories, Kyoto University, Yamashina, Kyoto, JAPAN \\ email: asai@kwasan.kyoto-u.ac.jp \\ ${ }^{2}$ Department of Earth and Planetary Science, University of Tokyo, Bunkyo, Tokyo, JAPAN \\ ${ }^{3}$ Nobeyama Radio Observatory, Minamisaku, Nagano, JAPAN \\ ${ }^{4}$ Solar-Terrestrial Environment Laboratory, Nagoya University, Toyokawa, Aichi, JAPAN
}

\begin{abstract}
We report a detailed examination about the relationship between the evolution of the $\mathrm{H} \alpha$ flare ribbons and the released magnetic energy during an X2.3 solar flare which occurred on 2001 April 10. We successfully evaluated the released energy quantitatively, based on the magnetic reconnection model. We measured the photospheric magnetic field strengths and the separation speeds of the fronts of the $\mathrm{H} \alpha$ flare ribbon, and estimated the released magnetic energy at the flare by using those values. Then, we compared the estimated energy release rates with the nonthermal behaviors observed in hard X-rays and microwaves. We also estimated the magnetic energy released during the flare. The estimated energy release rates in the $\mathrm{H} \alpha$ kernels associated with the hard X-ray sources are locally large enough to explain the difference between the spatial distribution of the $\mathrm{H} \alpha$ kernels and the hard X-ray sources. Furthermore, we reconstructed the peaks in the nonthermal emission by using the estimated energy release rates.
\end{abstract}

The large two-ribbon flare occurred at 05:10 UT, 2001 April 10 in NOAA 9415. The details of the flare were reported in several papers, e.g. in Asai et al. (2002, 2003) While we can see two-ribbon structure in $\mathrm{H} \alpha$ line center images observed with the Sartorius Telescope at Kwasan Observatory, Kyoto University, we see a few emission sources in hard X-ray (HXR) images obtained with Yohkoh/HXT. The difference between the spatial distributions of $\mathrm{H} \alpha$ kernels and those of HXR sources is probably caused by the low dynamic range of HXT (about 10). The HXR intensity which is emitted in bremsstrahlung is proportional to the number of accelerated electrons, and is thought to be proportional to the energy release rate, as Hudson (1991) and Wu et al. (1986) suggested. Therefore, if the energy releases at the HXR sources are large enough, that is, at least 10 times larger than the other sources, then the other sources are buried in noise. The nonthermal microwave synchrotron emission is also thought to be well correlated with the energy release rate.

Based on a 2D magnetic reconnection model, the energy release rate is written as the product of the Poynting flux carried into the reconnection region $S=2(4 \pi)^{-1} B_{c}^{2} v_{i}$ and the area of the reconnection region $A$, i.e. $d E / d t=S A$ (Isobe et al.2002), where $B_{c}$ is the coronal magnetic field strength and $v_{i}$ is the inflow velocity into the reconnection region. Since it is difficult to measure $B_{c}$ and $v_{i}$, we evaluate the energy release rate by using observable values, such as the magnetic field strengths at the photosphere $B_{p}$ measured with $S O H O / M D I$, and the separation speed of the $\mathrm{H} \alpha$ flare ribbons $v_{f}$. We estimated the reconnection rate $E$, which is defined as the reconnected magnetic flux per unit time, and expressed as $B_{c} v_{i}$. From the conservation of magnetic flux, it is farther rewritten as $B_{p} v_{f}$. We also estimated the Poynting flux $S$, by assuming that $B_{c}=a B_{p}$ $(a=$ constant $)$ and $S \propto a B_{p}^{2} v_{f}$. 

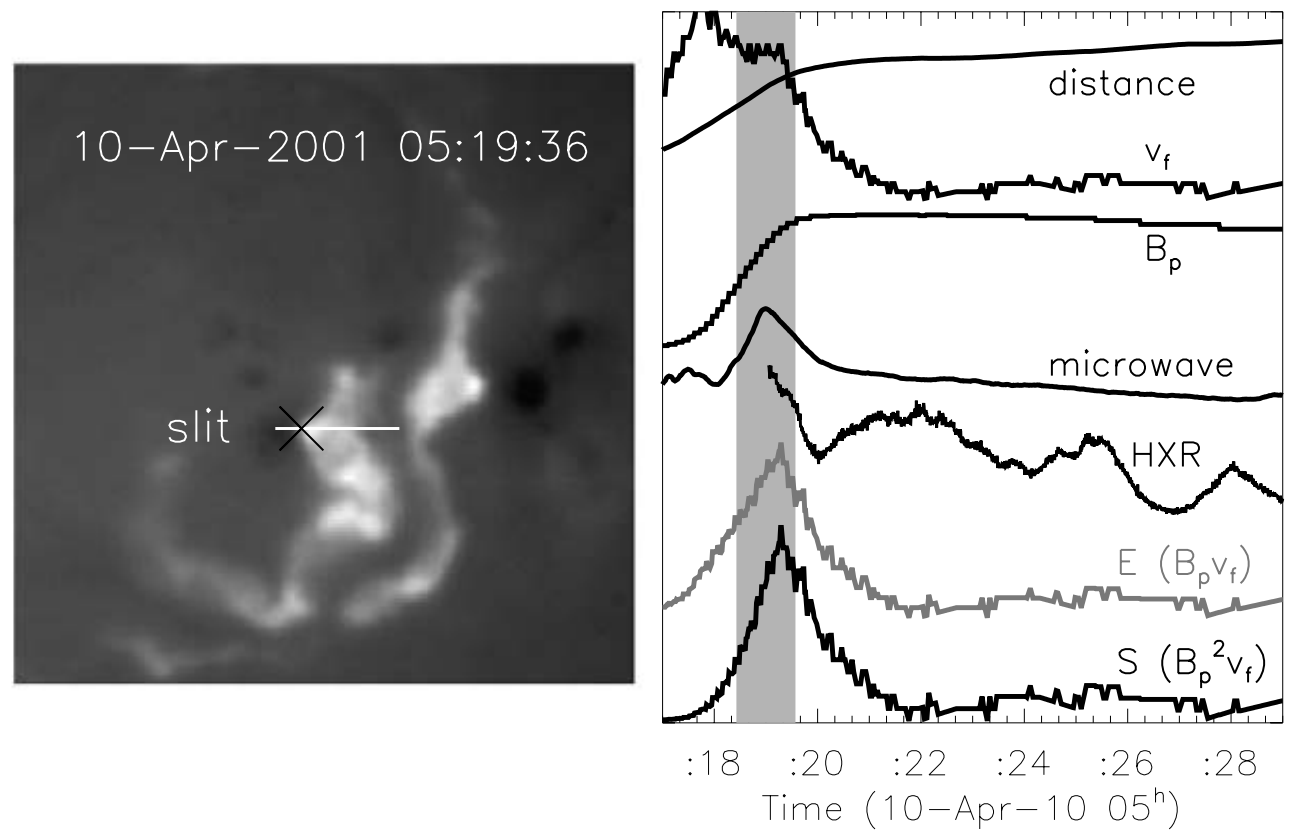

Figure 1. Left: An $\mathrm{H} \alpha$ image of the flare. The site of an HXR source is shown as cross. Right: Temporal evolutions of the flare ribbons and estimated energy release rates which are derived along the slit in the left panel. All are scaled arbitrarily. From top to bottom: Distance from the magnetic neutral line; separation velocity of the flare ribbon $\left(v_{f}\right)$; photospheric magnetic field strength at the ribbon-front $\left(B_{p}\right)$; radio correlation plot taken at $17 \mathrm{GHz}$ with NoRH; HXR count rate measured with HXT; reconnection rate $(E)$; Poynting flux $(S)$.

We measured $B_{p}$ and $v_{f}$ at the front of the $\mathrm{H} \alpha$ flare ribbon along the slits as shown in the left panel of figure 1 , and estimated the reconnection rate $E$ and the Poynting flux $S$. We compared them with the light curves in HXRs and microwaves. The right panel of figure 1 shows the time profiles of estimated $E$ and $S$, compared with the light curves in HXR and microwave obtained with HXT and with Nobeyama Radioheliograph (NoRH). The temporal evolutions of the physical values, such as $B_{p}, v_{f}$, and the distance of the front of the flare ribbons from the magnetic neutral line are also shown in the figure. The evolutions of $E$ and $S$ are well fitted with the HXR and microwave light curves, and they are enhanced largely enough at the HXR sources so that they can explain the difference between the spatial distribution of the HXR sources and that of the $\mathrm{H} \alpha$ kernels. We could reconstructed the peaks in the nonthermal emission. We also examined $E$ and $S$ at each radiation sources. $E$ and $S$ at the HXR sources are at least 16 times and 150 times larger than those at the $\mathrm{H} \alpha$ kernels without any HXR sources, respectively. They are enhanced largely enough at the HXR sources so that they can explain the difference between the spatial distribution of the HXR sources and that of the $\mathrm{H} \alpha$ kernels.

\section{References}

Asai, A., et al. 2002 Astrophys. J. 578, L91-L94.

Asai, A., et al. 2003 Astrophys. J. 586, 624-629.

Isobe, H. et al. 2002 Astrophys. J. 566, 528-538.

Hudson, H. S. 1991 Sol. Phys. 133, 357-369.

Wu, S. T. et al. 1986 In Energetic Phenomena on the Sun (eds. M. Kundu \& B. Woodgate) pp. 377. (NASA CP-2439). 